\title{
tecnologia tátil para a AVAliação da dor em Cegos
}

Ana Cláudia de Souza Toniolli ${ }^{1}$

Lorita Marlena Freitag Pagliuca ${ }^{2}$

Toniolli ACS, Pagliuca LMF. Tecnologia tátil para a avaliação da dor em cegos. Rev Latino-am Enfermagem 2003 março-abril; $11(2): 220-6$.

Trata-se de um estudo descritivo e exploratório das percepções dos cegos acerca do protótipo chamado Escala Tátil, para avaliação da intensidade da dor. Por meio de uma entrevista semi-estruturada, foram obtidos os relatos de cegos da Associação dos Cegos do Ceará, para descrever a natureza do protótipo, estabelecendo relações entre as suas experiências dolorosas e buscando significados para a sua utilização na cultura dos cegos. Dos relatos, foram identificados e analisados três temas: intensidade da dor na ponta dos dedos, percepção da escala tátil da dor, destacando-se textura, forma e tamanho, lapidando-se a Escala Tátil. A percepção sensorial tátil dos cegos gerou novos parâmetros de reflexão para o estudo da dor e direcionou a performance do protótipo para um processo de comunicação da dor mais satisfatório.

DESCRITORES: tecnologia, tato, avaliação, dor, cegueira

\section{TACTILE TECHNOLOGY FOR PAIN EVALUATION IN BLIND PEOPLE}

This is a descriptive study of blind people perceptions about a prototype called Tactile Scale for pain intensity evaluation. By means of a half-structured interview, reports were collected from blind people from the Ceará Blind People Association, to describe prototype nature, establishing relationships between their pain experiences and looking for meanings for their use in the blind people culture. Out of the reports, three issues were identified and analyzed: pain intensity at fingertips, pain tactile scale perception, highlighting texture, shape and size, and polishing the Tactile Scale. Blind people tactile sensory perception generated new reflection parameters to pain study and directed prototype performance to a more satisfactory pain communication process.

DESCRIPTORS: technology, touch, evaluation, pain, blindness

\section{TECNOLOGía tÁctil PARA LA EVALUACIÓN DEL dolor EN CIEgos}

Este es un estudio descriptivo de las percepciones de los ciegos acerca del prototipo llamado Escala Táctil para evaluación de la intensidad del dolor. A través de una entrevista semi-estructurada, se obtuvieron relatos de ciegos de la Asociación del Ciegos de la ciudad de Ceará-BR para describir la naturaleza del prototipo, estableciendo relaciones entre sus experiencias dolorosas y buscando significados para su utilización en la cultura de los ciegos. De los relatos fueron identificados y analizados tres temas: intensidad del dolor en la punta de los dedos, percepción de la escala táctil del dolor: textura, forma y tamaño y puliendo la Escala Táctil. La percepción sensorial de las personas ciegas generó nuevos parámetros de reflexión para el estudio del dolor y orientó la performance del prototipo hacia un proceso de comunicación del dolor más satisfactorio.

DESCRIPTORES: tecnología, tacto, evaluación, dolor, ceguera

\footnotetext{
${ }^{1}$ Mestre em Enfermagem Clínico-Cirúrgica. Doutoranda em Enfermagem, Bolsista da CAPES, Integrante do Projeto "Saúde Ocular", e-mail: acst@roadnet.com.br; ${ }^{2}$ Professor Titular do Departamento de Enfermagem, Coordenador do Projeto "Saúde Ocular" da UFC/CNPq. Universidade Federal do Ceará
} 
INTRODUÇÃO

$\boldsymbol{A}$ dor é uma experiência pessoal e complexa que envolve vários componentes sensoriais, afetivos, cognitivos, sociais e comportamentais. A multidimensionalidade de fatores relacionados a essa sintomatologia traz sérias implicações de ordem avaliativa pelos profissionais de saúde.

Para avaliá-la, é necessário desenvolver a compreensão do fenômeno, de forma subjetiva, dentro de uma visão holística e humanística, buscando a redução de comportamentos de dor, manifestos ou encobertos, e o aumento de comportamentos saudáveis. Uma avaliação acurada da dor permite compreender a origem e a magnitude do fenômeno, levando a um melhor manejo terapêutico ${ }^{(1)}$.

Mesmo existindo, à disposição dos profissionais de saúde, uma série de instrumentos clássicos para avaliar a dor, estes são pouco utilizados, talvez devido ao pouco conhecimento e/ou divulgação dessas tecnologias e pelas dificuldades de manejo que tornam ainda mais graves a subestimação e o subtratamento da dor.

As tecnologias criadas para avaliar o fenômeno doloroso evoluíram em sincronia com a evolução das teorias neurofisiológicas, que marcaram os limites das explicações dos mecanismos de modulação do fenômeno. Continua, portanto, a busca de instrumentos de avaliação da dor que possam fornecer dados passíveis de análises comparativas por meio de medidas escalonares e que ofereçam a possibilidade de expressão da qualidade da sensação, intercambiada pela sua multidimensionabilidade.

Por definição, a tecnologia proposta não envolve simplesmente a criação de um produto e avaliação dos seus impactos sobre a clientela. "É uma arte, um conhecimento dos instrumentos, isto é, tudo o que depende da sua elaboração, da sua criação, da justificação da sua utilização apropriada e da maneira de se servir deles"(2).

Diante desse contexto e sensibilizadas pela impotência de manter uma relação de ajuda, quando o ser que sente dor é possuidor de necessidades sensórias, busca-se, nesta pesquisa, re-construir o protótipo denominado "Escala Tátil da Dor", a partir da percepção de pessoas cegas.

A inteligência tátil e auditiva dos cegos mostra a possibilidade de comunicação, utilizando outras formas de linguagens. A pele registra mensagens ricas de valores, crenças e sentimentos e energias de experiências vividas desde a concepção. Dessa forma, criar tecnologia tátil para avaliar a intensidade da dor em pessoas cegas pode ser útil, também, para a clientela vidente, possuidora de recursos sensoriais tácteis.

\section{INSTRUMENTOS UNI E MULTIDIMENSIONAIS DA DOR}

Um instrumento de aferição da intensidade da dor determina quão forte é a dor para a pessoa que a experimenta e a eficácia da terapêutica utilizada. A terapia farmacológica poderá ser escolhida em função da intensidade da dor, em leve, moderada ou severa, em conformidade com o manejo da dor oncológica, preconizado pela Organização Mundial de Saúde (O.M.S.), chamado de "Escada analgésica da dor"(3-4), que pode ser utilizado, também, para o manejo da dor aguda.

A necessidade de precisão e controle da dor pelos profissionais de saúde reflete a busca, nas duas últimas décadas, de escalas alternativas para avaliar a intensidade da dor. Há dois tipos de instrumentos conhecidos para a mensuração da dor: os unidimensionais e os multidimensionais. Os primeiros são assim chamados por permitirem a mensuração de uma única dimensão da dor. A intensidade e os multidimensionais consideram duas ou mais dimensões.

Diversos autores confirmam a utilização dos instrumentos unidimensionais como predominantes até a década de 60 , pois acreditava-se, ainda, na experiência dolorosa como sendo o resultado da ativação direta de um sistema de projeção que servia de trajetória da periferia ao córtex cerebral, dependente exclusiva da área lesada ${ }^{(1,5-}$ 6)

Os nomes desses instrumentos eram determinados em função da natureza das categorias escolhidas. Quando numeradas, recebiam o nome de "Escalas Numéricas"; quando apresentavam visualmente adjetivos, chamavam-se "Escalas Verbais", e as "Analógicas-visuais" possibilitavam avaliações visuais. $\mathrm{O}$ auto-relato da dor pode ser classificado em três categorias de instrumentos: a "Escala Analógica-visual" (VAS) mensura uma dimensão da dor, o "Graphic Rating Scale" (GRS), o qual consiste em palavras distribuídas ao longo de um continuum de valores crescentes, e a "Numerical 
Rating Scale" (NRS), que utiliza uma reta numerada (Ex. $0-5$, ou 1-100), para refletir o aumento dos níveis da dor. Outros pesquisadores acrescentam a essa classificação a "Escala de Faces"(3,6-7).

Os instrumentos unidimensionais são mais freqüentemente utilizados para a mensuração da dor no pós-operatório e a respectiva efetividade da terapia analgésica. Embora possuam limitações, por simplificarem por demais a experiência dolorosa ${ }^{(1,6)}$, possibilitam momentos de aproximação entre o cuidador e o indivíduo com dor, podendo trazer indícios para uma compreensão subjetiva do fenômeno.

A partir das contribuições de Melsack, desde 1965, muito foi acrescentado para avaliação multidimensional da dor, principalmente com a criação do Questionário da Dor McGill (MQP), em 1975, outros instrumentos dessa natureza foram desenvolvidos, como o Wisconsin Brief Questionnaire $^{(8)}$ e o Memorial Pain Assesment Card ${ }^{(9)}$. São de extrema valia para a avaliação multidimensional da dor crônica e aguda, no entanto necessitam da compreensão cultural e cognitiva dos descritores, bem como adaptação do tempo a ser aplicado.

Alguns pesquisadores, ao aplicar o MQP na avaliação da dor, no pós-operatório, verificaram que os descritores mais freqüentemente utilizados para a expressão da dor pertenciam ao grupo sensorial ${ }^{(5,10)}$. Embora seja um fator de limitação do próprio questionário possuir um maior número de descritores sensoriais do que afetivos e avaliativos, pode-se refletir sobre a importância dada pelo ser humano às características espaciais de pressão, de tensão, térmica e de vivacidade da dor.

Além dos descritores da sensação, foram identificados descritores afetivos da expressão da dor no pós-operatório, ao julgar a intensidade percebida em relação a quanto o adjetivo definia a dor ${ }^{(11)}$. Com esse estudo, foi possível utilizar medições com o uso de métodos geradores de escalonamento de razão (estimação de magnitudes e emparelhamento intermodal) e avaliar quanto uma intensidade da dor foi maior ou menor do que outra.

Em nível sensorial, o processo comunicativo ocorre, também, por intermédio da pele, e o sistema tátil, por meio da multissensorialidade da pele, influenciando a mente ${ }^{(12)}$. Esses conceitos envolvem a interação humana e suas relações psicofisiológicas complexas, colocando em discussão outros elementos da sensorialidade tátil, como, por exemplo, a textura e a sua relação com a dimensão cognitiva e afetiva.

\section{A ESCALA TÁTIL DA DOR: MÉTODOS E PROCEDIMENTOS}

Trata-se de um estudo descritivo e exploratório para desenvolver tecnologia de comunicação não-visual para avaliar a dor, sendo que pesquisador e cegos agiram como co-participantes do processo, analisando e afinando o protótipo proposto.

A ACEC, local do estudo, é uma instituição sem fins lucrativos, com finalidades educacionais, culturais e profissionalizantes. Os seus associados são predominantemente pessoas cegas, com idade variada e predominante de adultos jovens.

O protótipo assemelha-se a uma escada em madeira, revestido de fórmica branca, com altura de 33 $\mathrm{cm}$ (330 mm), base de $11 \mathrm{~cm}(110 \mathrm{~mm})$, ápice de 1,5 cm. Possui 11 degraus, em formato tridimensional, sendo que cada degrau tem $3 \mathrm{~cm}(30 \mathrm{~mm})$. Não foi colocada numeração em Braille ou qualquer relevo ou textura diferente para cada degrau.

Para a proteção dos sujeitos da pesquisa, foram observados os aspectos éticos constantes na Resolução 196/96, Diretrizes e Normas Regulamentadoras de Pesquisa, envolvendo seres humanos ${ }^{(13)}$. Assim, a aprovação dos sujeitos (Diretoria das Associações/Cegos/ Familiares) aconteceu mediante a apresentação dos objetivos e métodos a serem utilizados, a garantia do anonimato das respostas e a utilização de instrumentos de coleta de dados, sem riscos para sua vida, em ambiente adequado.

Após o consentimento para a realização da pesquisa, no mês de junho de 2001, foi solicitado a cinco adultos cegos da ACEC descrever experiências dolorosas. Uma vez relatadas essas experiências, o pesquisador explicou o objetivo da Escala Tátil, entregando o objeto nas suas mãos, solicitando para avaliar, com uma nota de 0 a 10, cada uma das experiências. A menor proporção de massa representava nenhuma dor, a segunda tinha nota 1,0 e, assim, de forma contínua até a maior, que representava a dor máxima, com nota 10,0. Sem explicitação prévia da forma, tamanho ou textura, foi solicitada aos cegos a descrição da sua percepção sobre o instrumento utilizado. 
A duração de cada encontro foi de, aproximadamente, 30 minutos. Os relatos foram anotados pela pesquisadora, sendo codificados: E para "experts", e A para "alunos" com a respectiva numeração.

Para análise da intensidade da dor, as avaliações feitas com pontuações ordinais para cada degrau classificaram-se em dor: nenhuma (0), leve (1 a 2), moderada (3 a 5) e intensa (6 a 10). Essa numeração contínua existe na Escala Analógica Visual (EVA) e referese a centímetros ${ }^{(14-15)}$. No entanto, a Escala Tátil possui 30 milímetros em cada degrau ou nota avaliada e em todo o comprimento/altura 330 milímetros.

Os relatos foram decodificados e analisados por agrupamento temático, considerando o valor da sensorialidade tátil de análise dos cegos. A educação do olhar estético ${ }^{(16)}$ comparado à característica dessa análise traz a possibilidade de compreensão da análise realizada pelos cegos de descrever, construir, classificar, interpretar e re-criar.

A compreensão da percepção sensorial, afetiva e avaliativa do protótipo encontrou em elementos da comunicação visual ${ }^{(17)}$, a suposição de que, para haver comunicação visual, é necessário existir forma com conteúdo. Esse último é influenciado pela importância das partes constitutivas, como a cor, o tom, a textura, a dimensão, a proporção e suas relações compositivas com o significado. Sem esquecer do aroma e do paladar presentes, comumente, na vida do cego, a leitura tátil pode captar os estímulos existentes na cultura do cego da forma, do escalonamento e da textura.

\section{RE-CRIANDO A ESCALA TÁTIL DA DOR}

A Escala Tátil da Dor foi submetida à apreciação de cinco pessoas cegas, sendo três experts e dois alunos. Foram considerados experts pelo nível de escolaridade, pois três possuíam nível superior e, também, pelo fato de serem professores com experiência no ensino de pessoas cegas. Os dois alunos entrevistados eram adultos jovens e seguiam o curso supletivo na mesma escola.

Os relatos sobre a percepção da exploração tátil do protótipo de avaliação da dor permitiram a formulação de duas temáticas: a intensidade da dor na ponta dos dedos, percepção da escala tátil da dor, destacando textura, forma e tamanho e lapidando a Escala Tátil.
TEMA I - A intensidade da dor na ponta dos dedos

O resgate da experiência dolorosa vivenciada pelos cegos fez parte de um momento de sensibilização, o qual permitiu trazer, para o presente, o inconsciente sensorial e afetivo de cada um. Nesse momento, em harmonia com a tranqüilidade do ambiente e relaxamento, adentraramse no seu mundo interno, na busca de experiências dolorosas vividas, facilitando o processo de percepção do instrumento apresentado.

A expectativa de estar despertando o processo de abstração nos cegos, para a apreciação da estética da arte tecnológica proposta, corrobora com a afirmativa ${ }^{(16)}$ de que essas habilidades são processos que desenvolvem em contínuo, durante toda a vida, e parte, inicialmente, de uma visão egocêntrica e ingênua.

As experiências dolorosas referidas estavam relacionadas à tensão pré-menstrual, dor no pós-operatório de cirurgia cesariana e dores associadas às causas de cegueira: glaucoma e meningite. Houve mais de cinco relatos de experiências de dor. Isso porque, em alguns depoimentos, havia mais de um relato de dor referente a episódio de úlcera gástrica e dor no parto.

O relato da característica da dor é um dos indicadores considerados relevantes para a sua avaliação. Os cegos utilizaram adjetivos para expressar a dor mais marcante em frases como: (...) as dores eram fortes ( $E-1, E-$ 3). Tenho dores horriveis (E-2). (...) uma dor horrorosa (E-3).(..) nunca tive outra dor pior (A-1).(...) era insuportável, (...) uma angústia, (...) não sabia o que iria acontecer comigo (A-2).

$\mathrm{Na}$ busca de experiências dolorosas, alguns cegos fizeram comparações entre as dores sentidas e, de forma avaliativa, atribuíram adjetivos para qualificar a sua dor. Alguns adjetivos, relatados acima, assemelhamse aos descritores existentes no Inventário para a Avaliação da Dor, de McGill, como, por exemplo, os adjetivos "forte", "insuportável", que pertencem à dimensão avaliativa. Os adjetivos "horrível" e "horrorosa" não existem nesse Inventário. Expressam o sentimento experenciado no momento da dor, e poderiam constar na dimensão afetiva.

A cultura é um fator limitante do uso deste inventário. Para um país de tantos contrastes culturais, seria de extrema relevância estudar quais os adjetivos freqüentes e de uso popular utilizados no Ceará, que pudessem exprimir a dor.

Esse procedimento facilitou a utilização da Escala Tátil para avaliar quanto foi forte a sua dor, e os cegos 
compararam as dores experenciadas e atribuíram valores numéricos, de acordo com a solicitação da pesquisadora.

A experiência de dor no pós-parto e de parto foram consideradas intensas (nota 8,0) por duas mulheres cegas. A tensão pré-menstrual referida por uma cega (E2) obteve apreciação moderada (nota 5,0), diferente de outra que vivencia essa dor mensalmente, considerandoa como de intensidade leve, aferindo nota 1,0. Nota-se que a primeira não faz comparação com outros eventos dolorosos na vida, enquanto a segunda compara com episódios de úlcera gástrica e considera as dores associadas como intensas (nota 6,0). A nota máxima $(10,0)$, considerada como severa, foi referida nos processos dolorosos decorrentes das causas da cegueira: glaucoma e meningite.

A necessidade de qualificação e atribuição nominal de cada fenômeno doloroso vivido pelos cegos está presente em todos os relatos. Infere-se daí que a análise das notas atribuídas à intensidade da dor podem vir atreladas a categorias nominais qualificativas da cultura local. Além disso, podem mensurar valores ordinais, permitindo averiguar a possibilidade de realização de análise de variância e análise correlacional ou métodos considerados mais modernos ${ }^{(11)}$ como aqueles destinados a escalonamento de razão.

As experiências relatadas dos entrevistados foram representadas por apreciações de caráter avaliativo e afetivo de situações pessoais vividas. Há, nesse conjunto, o fenômeno de distração, susgestionabilidade, antecipação e referência à situações anteriores, vividas ou observadas, presentes na dimensão cognitiva $^{(18)}$.

As implicações emocionais e sociais estão imbricadas na percepção do estímulo doloroso e são capazes de modular a interpretação e a resposta à presença da dor. Existe um escore alto que representado, nas escalas unidimensionais, pela pessoa que sente dor, pode traduzir tanto um nível importante de dor, como também uma atitude, visando a enfatizar sua dor ou uma antecipação ansiosa, em face de uma evolução incerta, entre outros fatores ${ }^{(18)}$.

Nos relatos dos entrevistados há, inicialmente, atribuições qualitativas das experiências dolorosas e correlações entre as experiências vividas por alguns deles, para, em seguida, aplicar a nota. A complexidade do fenômeno doloroso impõe uma avaliação que permita a expressão qualificativa da dor e não somente quantitativa. Assim, as palavras-âncora "sem dor" e "dor máxima", sugeridas para o protótipo, pode recair no erro das escalas "Analógicas Visuais", de simplificarem por demais as experiências dolorosas e não permitirem operações estatísticas de nível mais elevado, pois geram dados apenas em nível ordinal e, no máximo, em nível intervalar.

A temática seguinte trata das percepções sensoriais dos experts e alunos da Escola de Cegos sobre a Escala Tátil da Dor.

TEMA II - PERCEPÇÃO DA ESCALA TÁTIL DA DOR: textura, forma e tamanho

A sensibilização afetiva e emocional das experiências dolorosas vividas despertou a percepção da dimensão estética, produzindo a criação de imagens mentais, na tentativa de encontrar relações estruturais.

Uma vez despertada a construção dos significados, inicia-se o interesse pelas propriedades formais do objeto de apreciação ${ }^{(16)}$. O julgamento da Escala Tátil pelos cegos é, então, intermediado pelos valores e padrões de conhecimento do cotidiano e pela objetividade da obra.

Para perceber a estética do objeto científico, os cegos utilizaram as pontas dos dedos e, pelo tato, buscaram a compreensão do objeto como um todo. Embora os julgamentos ocorram em função do seu mundo físico e moral, ainda estão presentes os sentimentos e associações pessoais. Nas falas dos cegos, foi possível identificar sentimentos associados às experiências dolorosas, embebidas de valores pessoais, dificultando a comparação com a Escala Tátil da Dor.

Com as pontas dos dedos, os cegos fragmentaram, mentalmente, a Escala Tátil da Dor, para compreender a relação entre a intensidade da dor sentida e a forma apresentada. Alguns elementos constitutivos das partes de um elemento visual, entre eles a dimensão, foram considerados relevantes para a compreensão da comunicação não-visual, principalmente ao relacioná-la com a finalidade da Escala, como se vê nas falas que se seguem: (...) o instrumento é muito bom. É grande. De bom tamanho! O cego precisa pegar tudo. O cego, quando fica doente, fica frágil e aí precisa pegar em tudo, ter uma visão global, (...) (A2).É tátil, resistente, de fácil explicação e entendimento (l-1).

A exploração dos objetos para o cego é tridimensional. Os objetos de tamanho pequeno ou médio possibilitam a preensão completa por meio das mãos, usando o tato, sendo mais bem decifrados do que objetos 
de maior volume ou massa ${ }^{(19)}$.

Os componentes visuais básicos para a compreensão da estrutura geral da "Escala Tátil", como uma forma de comunicação para as pessoas videntes, são diferentes para o cego. Apenas a forma, a direção, a textura, a dimensão, a escala e o movimento constituem elementos imprescindíveis, associados à percepção auditiva, gustativa e olfativa, para servir de conduto do processo comunicativo.

Pelo tato, os cegos procederam à análise do formato tridimensional da Escala, colocando-se satisfeitos com a justaposição de retângulos ao tatearem o instrumento, imaginando-o como (...) uma pirâmide! Imagino que é bonito (...).

Quanto à direção, houve preferência pelo processo de tatear de cima para baixo, (...) Gostei de contar de cima para baixo (l-1). A maior proporção em forma, dimensão e tamanho encontra-se na base da Escala, quando colocada na vertical, e o eixo diagonal exprime o contraste da composição do objeto, necessária às proporções variadas indicativas de diferentes intensidades de dor. O movimento da escala é evidenciado pelos cegos ao buscarem a direção e a dimensão que determinaram a estética singular do objeto.

Os cegos sugeriram que fosse acrescido na Escala (...) algo para destacar as notas, (...) aspereza. (A-2) ou (...) um tipo de lixa para cada nota, começando pela mais fina (I-2), sendo que (...) o áspero poderia está na dor grande (I-2). A textura, na percepção tátil dos cegos, é fator essencial para o entendimento da existência de nuances ou diferença no objeto apreciado. A composição de uma substância relaciona-se com a textura, por meio de variações mínimas na superfície do material. Com ela, o contraste faz sentido, pois há uma necessidade humana de organizar os estímulos em totalidades racionais ${ }^{(17)}$.

A noção de contraste poderia oferecer outros atributos, além dos valores ordinais. As sensações oriundas de texturas diferentes, mediadas por um valor subjetivo, podem dar uma melhor idéia de proporção, direção e de intensidades diferentes.

A percepção tátil dos cegos, tão inteligentemente trabalhada e essencial para o seu contato com o mundo, permitiu aquilatar a proporção entre os degraus, destacando a importância disso para estabelecer acordo com a finalidade do objeto.
Há métodos sendo utilizados, modernos e precisos, usando a teoria de Medidas e métodos escalares, de Stevens, para escalonar e distingüir as dimensões sensoriais e afetivas da dor ${ }^{(20)}$.

O critério da mensurabilidade da Escala Tátil pode ser ajustado, adaptando-se adjetivos qualificativos da dor, milimetrando-se, com orifícios, a escala por degraus e por comprimento. Além disso, o manejo tátil das pessoas cegas pode facilitar a utilização de algo que preencha o orifício, no momento de avaliar o adjetivo escolhido.

A mensuração da escala tátil possibilita uma estimação estatística, à primeira vista, de nível ordinal e intervalar, tanto como as escalas consideradas categóricas, como a Escala Analógica Visual ${ }^{(14)}$. No entanto, o processo estatístico será considerado, para uma amostra maior, passível de uma melhor análise.

A utilização de texturas diferentes para indicar notas diferentes foi sugerida pelos cegos para identificar, na dor mais intensa, uma textura mais áspera, e, na dor mais fraca, uma textura mais fina. O conhecimento dos experts acerca do mundo tátil foi de essencial importância para lembrar a existência de (...) dez tipos diferentes de lixas no comércio (I-2), pontuando, assim, cada nota e deixando uma textura lisa para o caso de não sentir dor. Além disso, sugeriram outras utilidades, do tipo pedagógico, inclusive com crianças e na clínica da dor, para videntes e nãovidentes.

\section{REFLEXÕES FINAIS}

De modo geral, o protótipo permitiu a avaliação da intensidade da dor em pessoas cegas, apesar do número limitado de pessoas. Contudo, considerando o objetivo de aperfeiçoá-lo, em co-participação com os cegos, é factível o design de um novo protótipo com as nuances sugeridas, de textura, de tamanho e de proporção.

A unidimensionalidade pode dar lugar, no novo protótipo, a outras dimensões de fatores relacionados à dor, ao serem adicionados adjetivos capazes de descrever a experiência dolorosa em estreita relação com as texturas. Com um número mais significativo de cegos, será possível estimar correlações e possibilidades estatísticas de escalonamento de razão entre as intensidades e categorias de dor. 


\section{REFERÊNCIAS BIBLIOGRÁFICAS}

1. Pereira LV, Faleiros Sousa FAE. Mensuração e Avaliação da dor pós-operatória: uma breve revisão. Rev Latino-am Enfermagem 1998 julho; 6(3):77-84.

2. Colliére MF. Promover à vida: da prática das mulheres de virtude aos cuidados de enfermagem. Damaia/Lisboa: Printipo; 1989.

3. Couturier M. La Douleur: place des antalgiques. $2^{a}$ ed. França: UPSA Laboratoires; 1996.

4. Organização Mundial de Saúde. Methods of Assessement of avoilable blindness. Genebra; 1982.

5. Melzack R, Abbott FV, Zackon W, Mulder DS, Davis MW. Pain on a surgical ward: a survey of the duration and intensity of pain and the effectiveness of medication. Pain 1987 January; 29(0):67-72.

6. Tollison CD. Definitions of the Pain. In: Tollison CD. Handbook of Pain Management. $2^{a}$ ed. Massachussets (EUA): Mosby; 1994.

7. Bonnet F A. A dor no Meio Cirúrgico. Porto Alegre: Artes Médicas; 1993.

8. Daut TL, Cleeland CS, Flanery RC. Development of the Wisconsin brief pain questionnary to asses pain in cancer and another disease. Pain 1983; 17:197-210.

9. Fishman B, Pasternak S, Wallenstein SL, Houde RW, Holland JC, Foley KM. The memorial pain assessment card. Cancer 1987; 60:1151-8.

10. Kim HS, Schwartz-Barcott D, Holter IM, Lorensen M. Developing a translation of the McGill pain questionnaire for cross-cultural comparision: an example from Norway. J Adv Nurs 1995 January; 21:421-6.

11. Faleiros Sousa FAE, Silva JA. Uso e Aplicação da metodologia psicofísica na pesquisa em enfermagem. Rev Latino-am Enfermagem 1996 maio; 4(2):147-78.

12. Montagu A. Tocar: o significado humano da pele. $4^{\mathrm{a}} \mathrm{ed}$. São Paulo (SP): Summus; 1988.

13. Ministério da Saúde (BR). Resolução n.1, de 13 de junho de 1998. Normas de pesquisa em saúde. Brasília (DF): Ministério da Saúde; 1998.

14. Huskisson EC. Measurement of pain. Lancet 1974; 2(7889):1127-31.

15. Ready LB, Edwards WT. Tratamento da dor aguda. Rio de Janeiro (RJ): Revinter; 1995.

16. Rossi MHW. A compreensão do desenvolvimento estético. In: Pillar AD. organizadora. A educação do olhar no ensino das artes. Porto Alegre (RS): Mediação; 1999. p. 23-36.

17. Dondis AD. Sintaxe da Linguagem Visual. $2^{a}$ ed. São Paulo: Martins Fontes; 1997.

18. Cambier J, Masson M, Dehen H. Semiologia da Sensibilidade. In: Cambier J, Masson M, Dehen H. Manual de Neurologia. 9a ed. Rio de Janeiro: Medsi; 1999. p. 1-30.

19. Pagliuca LMF. A Arte na ponta dos dedos: a pessoa cega. Rev Latino-am Enfermagem 1997 abril; 2(4):127-37.

20. Faleiros Sousa FAE. Psicofísica da Dor. $4^{\circ}$ Simpósio Brasileiro e Encontro Internacional sobre Dor; 1999. junho 10-13; São Paulo, p. 144-7. 